\title{
REVISIÓN SISTEMÁTICA DEL ESTADO DEL CONOCIMIENTO DE los Vertebrados del PARQUe NACIONAL Podocarpus
}

\author{
SYSTEMATIC REVIEW OF THE STATE ABOUT THE KNOWLEDGE OF THE \\ Vertebrates of the Podocarpus National PARK \\ Leonardo Ordóñez-Delgado*1 ${ }^{*}$, Claudia Ramón-Vivanco ${ }^{2}$ (:) y Valeria \\ Ortiz-Chalan ${ }^{3}$ (i)
}

\author{
${ }^{1}$ Laboratorio de Ecología Tropical y Servicios Ecosistémicos - EcoSs Lab. Universidad Técnica Particular de Loja, Departamento \\ de Ciencias Biológicas, Calle París, San Cayetano Alto, Loja, Ecuador \\ ${ }^{2}$ Grupo de Investigation Planning of Land and Social Systems - PLANOSS. Universidad Técnica Particular de Loja, Departa- \\ mento de Ciencias Biológicas, Calle París, San Cayetano Alto, Loja, Ecuador \\ ${ }^{3}$ Titulación en Gestión Ambiental. Universidad Técnica Particular de Loja, Departamento de Ciencias Biológicas, Calle París, \\ San Cayetano Alto, Loja, Ecuador
}

*Autor para correspondencia: lyordonez2@utpl.edu.ec

Manuscrito recibido el 12 de enero de 2019. Aceptado, tras revisión, el 24 de abril de 2019. Publicado el 1 de septiembre de 2019.

\section{Resumen}

El Parque Nacional Podocarpus es una de las áreas protegidas de mayor importancia y tamaño de los Andes Tropicales del sur de Ecuador. Desde hace mucho tiempo esta reserva ha sido el centro de atención de un importante número de investigadores que, entre otros elementos, han tratado de explicar diversos tópicos relacionados a la fauna que alberga. Sin embargo, hasta el año 2018 no se contaba con una sistematización adecuada de estas investigaciones. Con el objetivo de establecer el nivel de conocimiento alcanzado sobre los vertebrados del área protegida, se estructuró un proceso metodológico para la recopilación, sistematización y análisis de la información existente sobre esta temática. Se generó una base de datos de los estudios recopilados, con un total de 128 trabajos: 64 sobre aves, 26 de mamíferos, 22 sobre anfibios, 6 sobre reptiles, 2 sobre peces y 8 investigaciones que abarcan más de un grupo faunístico al mismo tiempo. El período con la mayor cantidad de publicaciones corresponde a la década del 2000. De las 16 localidades identificadas en donde se han efectuado trabajos sobre los vertebrados de esta área protegida, destacan con el mayor número de investigaciones Tapichalaca y Cajanuma, con 33 y 24 estudios, respectivamente. Esta información constituye la primera aproximación respecto del nivel de investigación alcanzado sobre los vertebrados que mantiene y protege este parque nacional.

Palabras clave: Fauna, aves, anfibios, reptiles, mamíferos, peces, Parque Nacional Podocarpus, Ecuador. 


\begin{abstract}
The Podocarpus National Park is one of the most important and biggest protected areas of the Tropical Andes of southern Ecuador. This reserve has been the center of attention for a large number of researchers who, among other elements, have tried to elucidate various topics related to its fauna. However, there has not been adequate systematization of these investigations so far. In order to establish the level of knowledge reached on the vertebrates of the protected area, a methodological process was structured for the compilation, systematization and analysis of existing information on this subject. A database of the collected studies was generated with a total of 128 works: 64 on birds, 26 on mammals, 22 on amphibians, 6 on reptiles, 2 on fish and 8 research works involving more than one faunistic group at the same time. The period with the largest number of publications corresponds to the decade of the 2000. Among the 16 locations identified where work has been done on the vertebrates of this protected area stand out Tapichalaca and Cajanuma with the largest number of investigations, with 33 and 24 studies, respectively. This information constitutes the first approximation regarding the level of research achieved on vertebrates that maintains and protects this national park.
\end{abstract}

Keywords: Fauna, birds, amphibians, reptiles, mammals, fish, Podocarpus National Park, Ecuador.

Forma sugerida de citar: $\quad$ Ordóñez-Delgado, L., Ramón-Vivanco, C. y Ortiz-Chalan, V. (2019). Revisión sistemática del estado del conocimiento de los Vertebrados del Parque Nacional Podocarpus. La Granja: Revista de Ciencias de la Vida. Vol. 30(2):7-18. http://doi.org/10.17163/lgr. n30.2019.01.

IDs Orcid:

Leonardo Ordóñez Delgado: https:/ /orcid.org/0000-0002-4593-1728

Claudia Ramón Vivanco: https:/ / orcid.org/0000-0002-6119-238X

Valeria Ortiz Chalan: https:/ /orcid.org/0000-0003-2160-0964 


\section{Introducción}

A pesar de su pequeña superficie $\left(283561 \mathrm{~km}^{2}\right)$, Ecuador se considera un referente en el tema de biodiversidad, debido a que su riqueza de especies, ecosistemas y elevados niveles de endemismo, entre otros factores, han influido para que el país sea reconocido como una de las naciones megadiversas (Mittermeier, 1997). Ecuador alberga al menos 4718 especies de vertebrados, entre ellos, 436 especies de mamíferos (Tirira, 2018), 1626 especies de aves (Freile y Restall, 2018), 609 especies de anfibios (Ron y col., 2019), 473 especies de reptiles (TorresCarvajal, Pazmiño-Otamendi y Salazar-Valenzuela, 2018), 635 especies de peces dulceacuícolas, y 939 marinos y estuarinos (Jiménez-Prado, 2010). Además, más de 17748 especies de plantas vasculares se han reportado en el territorio nacional (Neill, 2012). Pero, a pesar de esta destacada realidad, el 36,25\% de las especies del país en el 2014 se consideraron amenazadas, ubicando a Ecuador en el primer lugar en cuanto a los países con especies amenazadas de Sudamérica (UICN, 2014).

Entre las estrategias que el Estado ecuatoriano ha empleado para enfrentar esta problemática está la creación y gestión de una red de áreas protegidas públicas, privadas y comunitarias, amparadas en la Constitución de la República (Constituyente, 2008). Las áreas protegidas se definen como la estrategia de conservación por excelencia (Primack y col., 1998; Dudley y Stolton, 2010; Watson, Dudley y Hockings, 2014) las mismas han evolucionado desde una visión exclusiva de conservación de biodiversidad a objetivos más diversos, entre ellos la provisión de beneficios sociales y económicos (Watson, Dudley y Hockings, 2014).

El Parque Nacional Podocarpus (PNP), localizado entre las provincias de Loja y Zamora Chinchipe, es una de estas áreas destinadas a la conservación de la biodiversidad y mantenimiento de los servicios ambientales de la región sur del país (Apolo, 2002; Calderón, 2002). Este Parque Nacional es parte del "hotspot" Andes Tropicales, el más rico en biodiversidad en el planeta (Myers y col., 2000) y de la Ecorregión Terrestre Prioritaria "Páramos de la Cordillera Central" (Dinerstein y col., 1995), ecorregión presente en Ecuador exclusivamente en los Andes del Sur, la misma que está conformada por una suerte de islas pequeñas de ecosistemas altoandinos, confinados a los picos de las montañas centrales e internudos de los Andes por sobre los 3000 msnm (Cuesta y col., 2005).

Además, esta área protegida se ubica dentro de la depresión de Huancabamba, la barrera biogeográfica más importante de los Andes para la distribución de especies en sentido norte sur (Duellman, 1979; Cuesta y col., 2005; Ordóñez-Delgado, 2011). De ahí que se considere a este sitio como un centro de endemismo de plantas denominado Región de Huancabamba (Davis y col., 1997). Cuesta y col., (2005) proponen que es a este elemento geográfico al que se puede atribuir la baja similitud en la composición de flora y fauna de esta zona, respecto de los páramos de los Andes ubicados hacia el norte. Por otra parte, en el sitio en donde se localiza el PNP se sobreponen los centros de endemismo de los Andes del Norte y de Tumbes (Terborgh y Winter, 1983) y confluyen dos corrientes bioclimáticas, una proveniente de la Amazonía con grandes cantidades de humedad, y la otra del Pacífico, con la influencia de los vientos secos provenientes del norte de Perú (Ordóñez-Delgado, 2011).

Todas estas condiciones, sumadas a su irregular orografía, han influido para que se presenten en su interior varios microclimas, hábitats y nichos, dando como resultado una gran diversidad de flora y fauna, además de significativos niveles de endemismo (Cuesta y col., 2005; Ordóñez-Delgado, 2011). Esta área protegida constituye el eje de los esfuerzos de conservación en los Andes del Sur del país, entre otros, por el aporte en servicios ambientales a la región (Apolo, 2002). Del interior del área protegida nacen tres cuencas hidrográficas binacionales: Catamayo-Chira, Mayo-Chinchipe y Zamora; en su interior se localiza el sistema lacustre Lagunas del Compadre, reconocido como un sitio Ramsar (Humedal de Importancia Internacional) (OrdóñezDelgado, 2011) y se constituye en el área núcleo de mayor tamaño de la Reserva de Biósfera Podocarpus El Cóndor, reconocida en el 2007 por la UNESCO (Serrano, 2008).

Son todos estos elementos los que han influido, desde hace mucho tiempo, para que la comunidad científica nacional e internacional haya mostrado interés en el desarrollo de estudios al interior de este territorio (Aguirre y col., 2002; Serrano, 2008). Sin embargo, las investigaciones efectuadas en el área protegida no han contado hasta el momento con un adecuado proceso de análisis y sistematización, al grado de desconocer el nivel que han alcanzado 
hasta la actualidad o la dinámica experimentada a lo largo de su historia. Uno de los pasos iniciales para definir cuanto se conoce o desconoce de un tema dado es lo que se define como el "análisis del estado del conocimiento" que se conceptualiza como "el análisis sistemático y la valoración del conocimiento generado, en torno a un campo de investigación, durante un periodo definido" (Rueda, 2003).
El presente trabajo se fundamenta en este concepto y se orienta a establecer el "estado del conocimiento" existente hasta el momento sobre los vertebrados que alberga esta área protegida, las temáticas que en torno a este grupo de fauna se han investigado, la ubicación geográfica de los estudios, los vacíos de información existentes y las líneas de investigación prioritarias a futuro.

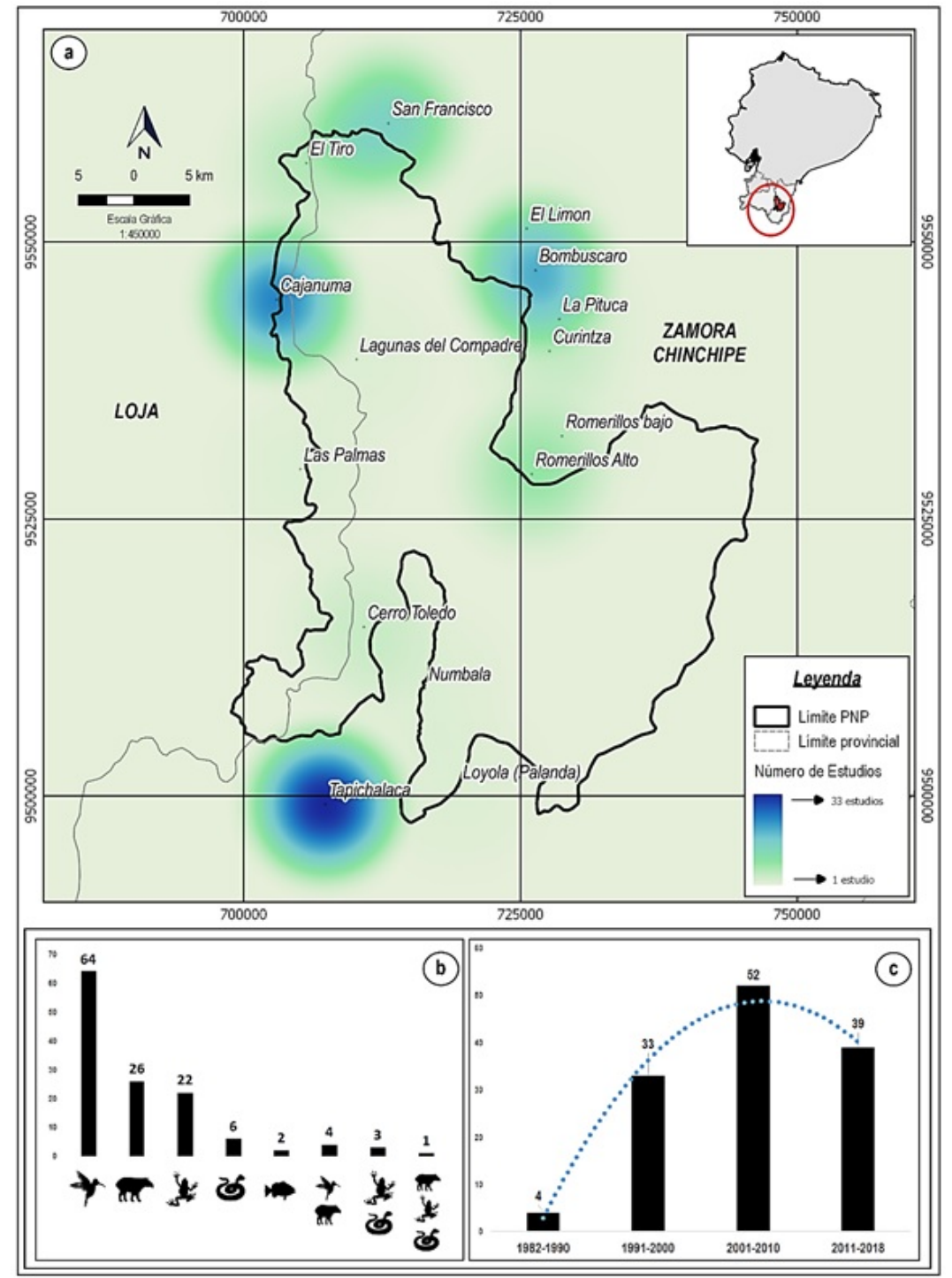

Figura 1. a) Representación gráfica del nivel de influencia geográfica de los estudios sobre vertebrados del Parque Nacional Podocarpus. b) Número de estudios por taxa. c) Número de estudios por rango temporal a partir de la declaratoria del área protegida. 


\section{Materiales y método}

\section{1 Área de Estudio}

El Parque Nacional Podocarpus (PNP) se ubica entre Numbala y el Nudo de Sabanilla, en el límite fronterizo de las provincias de Loja y Zamora Chinchipe en el sur de Ecuador (Ordóñez-Delgado, 2011). Comprende una superficie de 146280 ha y fue declarado oficialmente el 15 de diciembre de 1982 (Acuerdo Ministerial $N^{\circ}$ 0398, Registro Oficial. $N^{\circ} 404,5$ de enero de 1983) (Ministerio de Agricultura, Ganadería y Pesca de Ecuador, 1983). Aproximadamente el $83 \%$ del territorio de esta área protegida forma parte de la provincia de Zamora Chinchipe y el17\% a la provincia de Loja. Su rango altitudinal se distribuye entre los 900 y 3650 msnm (Aguirre y col., 2002). La temperatura del sector fluctúa entre 20 y $25{ }^{\circ} \mathrm{C}$ en la zona baja (amazónica) y de 8 a $15^{\circ} \mathrm{C}$ en la zona alto andina (Maldonado y Numa, 2002) (Figura 1a).

\subsection{Métodos}

El proceso de recopilación, análisis y sistematización de la información contempló los siguientes pasos: El área de trabajo para el análisis abarcó todo el parque nacional, considerando para esto sus límites oficiales. Sin embargo, debido a que varias localidades investigadas se encuentran inmediatamente fuera de los límites de la misma, se consideró ampliar el análisis a la zona de influencia inmediata del área. Se efectuó la búsqueda, recopilación, análisis y sistematización de la información sobre los vertebrados, en fuentes primarias y secundarias. Como fuentes primarias, se consideró la literatura científica, entre estas, publicaciones indexadas o con revisión por pares, además de libros con ISBN; literatura gris, que consideró a libros o documentos sin ISBN pero con aval científico (es decir documentos de investigadores reconocidos en el ámbito de trabajo del área protegida y la temática tratada en este documento), además de tesis de pregrado y posgrado de universidades que poseen repositorios de estas investigaciones en línea. Como fuentes de información secundaria se contempló el desarrollo de entrevistas realizadas a investigadores y entidades vinculadas al área protegida, entre estos: docentes de la Universidad Técnica Particular de Loja, Universidad Nacional de Loja y/o investigadores de ONG y entidades de investigación que han realiza- do estudios sobre el Parque Nacional Podocarpus y sus alrededores. Finalmente, se tuvo acceso a la base de datos de permisos de investigaciones científicas manejada por la Coordinación Zonal 7 del Ministerio del Ambiente de Ecuador para esta área protegida.

Para la búsqueda de información en bases de datos científicas, así como en repositorios bibliográficos y revistas indexadas, se establecieron un conjunto de palabras clave en español e inglés. Luego de establecer las palabras clave, se utilizaron otros parámetros de optimización de búsqueda como, por ejemplo:

- Poner entre comillas ("“") las palabras clave de búsqueda, para buscar una frase exacta.

- Usar el signo más (+) delante de las palabras del tema que se va investigar, para que todas estas sean tomadas en cuenta en la búsqueda en la web.

- Usar los operadores "OR" y "AND" en mayúsculas entre dos palabras clave para encontrar páginas que contengan alguna de estas en su texto.

- Usar el comando "Allintitle" seguido de las palabras clave el cual permitió encontrar documentos con estas palabras en su título.

- Usar el comando "Allintext" antes de las palabras clave para encontrar investigaciones que en su texto incluyan las palabras especificadas para la búsqueda.

Entre las palabras que se utilizaron para la búsqueda de información constan las siguientes: Parque Nacional Podocarpus Fauna del Parque Nacional Podocarpus Aves (mamíferos, anfibios, reptiles, peces) del Parque Nacional Podocarpus Vertebrados del Parque Nacional Podocarpus Vertebrados del Podocarpus Nombre de las localidades: Cajanuma, Tapichalaca, Bombuscaro, San Francisco, Cerro Toledo, Numbala, Vilcabamba, por si solas, o sumadas al nombre del área protegida: Parque Nacional Podocarpus. Aves (o el nombre de cada grupo faunístico) más el nombre de las localidades descritas en el párrafo previo, más el nombre del área protegida.

Se consideró utilizar estos últimos nombres por cuanto son los sectores de acceso más importantes 
al área protegida. Todas estas frases o palabras (salvo los nombres propios), fueron traducidas al inglés para ampliar la búsqueda a ese idioma. Con los parámetros mencionados se buscaron documentos en bases de datos y repositorios científicos tales como: Academia.edu, BioOne, Biodiversity Heritage Library, BirdLife International, Fauna Web Ecuador, Google Schoolar, ResearchGate, SciELO, Science Direct, Scopus, Semantic Scholar, Web of Science. Por otra parte, se buscó en las bases de datos de tesis de pregrado y postgrado de las siguientes universidades: Universidad Técnica Particular de Loja, Universidad Nacional de Loja, Pontificia Universidad Católica del Ecuador, Universidad Central del Ecuador y Universidad del Azuay.

Un método adicional de búsqueda de información utilizado fue el tomar las referencias bibliográficas de los documentos recopilados como punto de partida para búsqueda de documentos de interés particular. Si alguna de estas referencias hacía mención a estudios realizados sobre vertebrados en el PNP y su zona de influencia inmediata, se procedió a la búsqueda del documento completo en la web u otra fuente de información.

Con la información obtenida se elaboró una matriz en Excel, que sirvió como base de datos para el análisis de la información. Esta matriz estaba conformada por los siguientes ítems: Número del estudio, grupo faunístico, autor/a/es/as, título, fecha de publicación, tipo de documento (artículo, tesis, libro, informe), área de conocimiento (ecología, diversidad, biogeografía, conservación, taxonomía), localidad de estudio, coordenadas y altitudes referenciales. Para documentos que omitían esta última información se definieron coordenadas referenciales de localidades cercanas a la localidad de estudio, por medio de la revisión detallada de la información mencionada en materiales y métodos de cada documento o consulta a expertos.

Con la información recopilada se cuantificaron el número total de estudios, número de estudios por grupo faunístico, publicaciones por año, distribución geográfica y por pisos zoogeográficos. Los estudios que consideraban más de un grupo faunístico en la investigación, o dos o más localidades de estudio, fueron contabilizados por separado; es decir, si un estudio abarcaba aves y murciélagos, se contabiliza como un estudio multitaxa aparte, más no se contabiliza como un estudio de aves y uno de murciélagos, esto con la finalidad de evitar que el resultado del número de investigaciones se sobredimensione.

El análisis temporal de los estudios se efectuó por décadas, a partir de la creación del área protegida (1983), hasta diciembre de 2018. Para la distribución de los estudios respecto de los pisos zoogeográficos del Ecuador, mismos que corresponden a una clasificación general de la fauna del país conforme a su distribución altitudinal, se utilizó la propuesta de Albuja y col., (2012). De ahí que, conforme al rango altitudinal presente en el área protegida, se trabajaron con los siguientes pisos zoogeográficos: Subtropical oriental (1000 a $2000 \mathrm{msnm}$ ), templado (2000 a $3000 \mathrm{msnm}$ ) y altoandino (>3000 msnm).

Utilizando el programa QGIS (QGIS, 2018), se aplicó el estilo de simbolización de "mapa de calor" a las localidades georreferenciadas en donde se realizaron los estudios de fauna del PNP, utilizando la ponderación por puntos que contiene el número de estudios realizados en cada sitio, para determinar la intensidad del mapa. Se utilizó una gama de colores que va de verde (menor intensidad) a azul (mayor intensidad). Cabe recalcar que para la generación de este mapa no se tomó en cuenta la distinción de la taxa estudiada. 


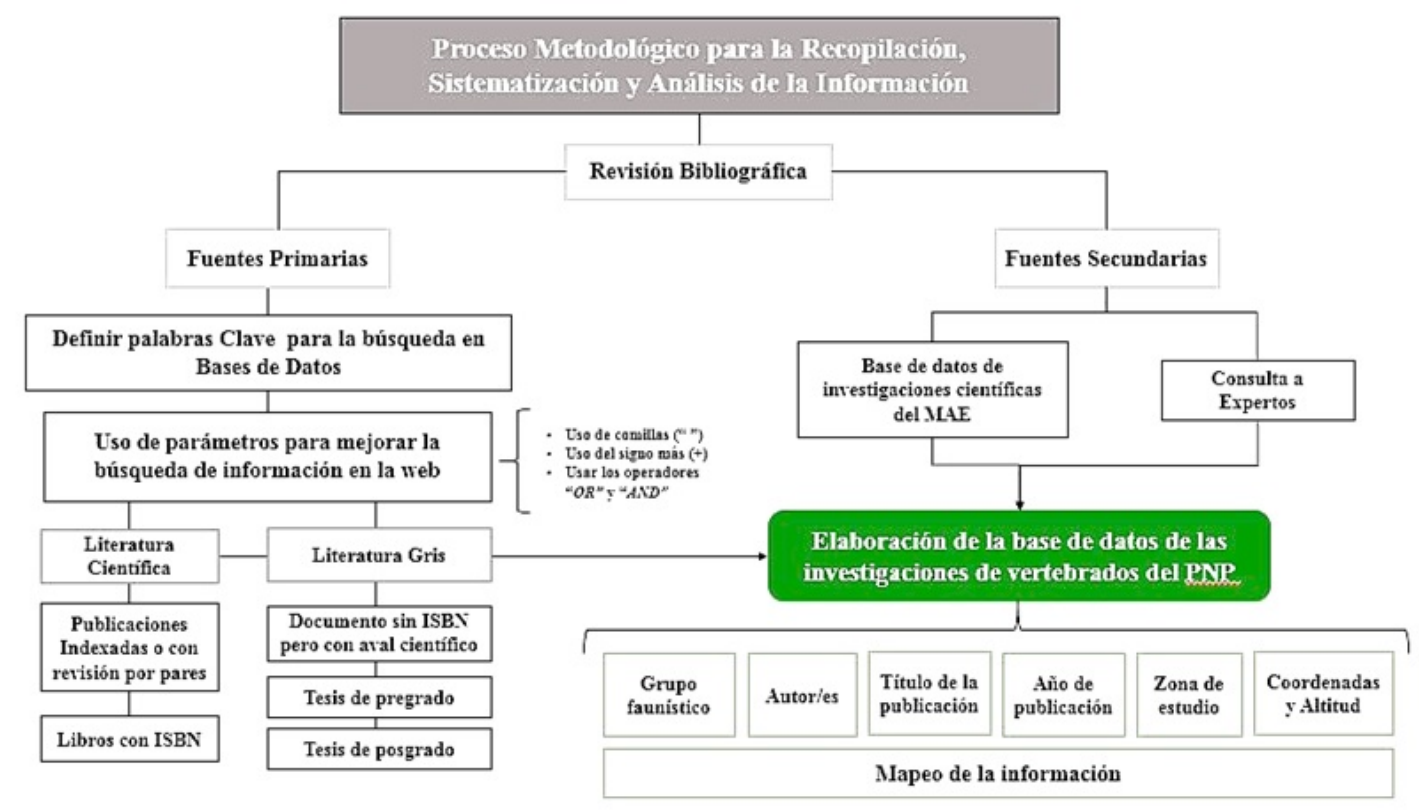

Figura 2. Esquema del proceso metodológico desarrollado para el análisis sistemático del estado de conocimiento de los vertebrados del Parque Nacional Podocarpus.

\section{Resultados}

\subsection{Esquema metodológico desarrollado}

El primer resultado del presente trabajo lo constituye la propuesta metodológica para la recopilación, análisis y sistematización de la información. A continuación, se propone el esquema gráfico resultante del proceso desarrollado (Figura 2).

\subsection{Número de estudios}

Se recopilaron 128 publicaciones sobre vertebrados del Parque Nacional Podocarpus y su zona de amortiguamiento. De estas, $84(66 \%)$ corresponden a publicaciones científicas y $44(34 \%)$ corresponden a literatura gris. Del total, 64 corresponden a aves (50\%), 26 a mamíferos (20\%), 22 a anfibios (17\%), 6 a reptiles $(5 \%)$ y 2 a peces $(2 \%)$. Se encontraron ocho estudios multitaxa: cuatro, sobre aves y mamíferos $(3 \%)$, tres sobre anfibios y reptiles $(2 \%)$; y, un estudio de mamíferos, anfibios y reptiles (1\%) (Figura $1 b)$.

\subsection{Número de publicaciones por rango temporal}

Respecto del rango temporal, la década inicial de creación del área protegida (1982-1990) constituye el periodo con menor número de publicaciones, mismas que en las siguientes décadas se incrementan significativamente. Sin embargo, en la década actual se evidencia un decrecimiento de al menos el $25 \%$ en cuanto al número de investigaciones realizadas en esta región sobre los vertebrados que alberga (Figura 1c).

\subsection{Distribución geográfica de los estudios}

En base al análisis de la información recopilada, se identificaron 16 localidades estudiadas para el área protegida, 15 corresponden a sitios individuales y una asignada a los estudios que abarcan a toda el área protegida. De las 128 publicaciones identificadas, $89(69 \%)$ corresponden a publicaciones que abarcan una sola localidad de estudio, $10(8 \%)$ a trabajos que abarcaron dos localidades, $10(8 \%)$ a estudios con tres localidades (8\%); y, 19 (15\%) a trabajos que se plantean como investigaciones que abarcan la totalidad del PNP (Figura 1a). Sin embargo, si se contabilizan el número de investigaciones de 
las cuales ha sido objeto cada localidad que forman parte de las 128 publicaciones mencionadas previamente, destacan Tapichalaca $(n=33)$ y Cajanuma ( $n=24)$ como los sitios con mayor número de investigaciones efectuadas. Le siguen los estudios que abarcan en su descripción a toda el área protegida PNP ( $n=19)$, luego Bombuscaro $(n=18)$, San Francisco $(n=14)$ y Romerillos Alto $(n=7)$. Mientras que el resto de localidades han sido objeto de trabajo de cinco procesos de investigación o menos.

\subsection{Número de publicaciones por piso zoo- geográfico}

Respecto de la distribución de los estudios en base a la clasificación de los pisos zoogeográficos del

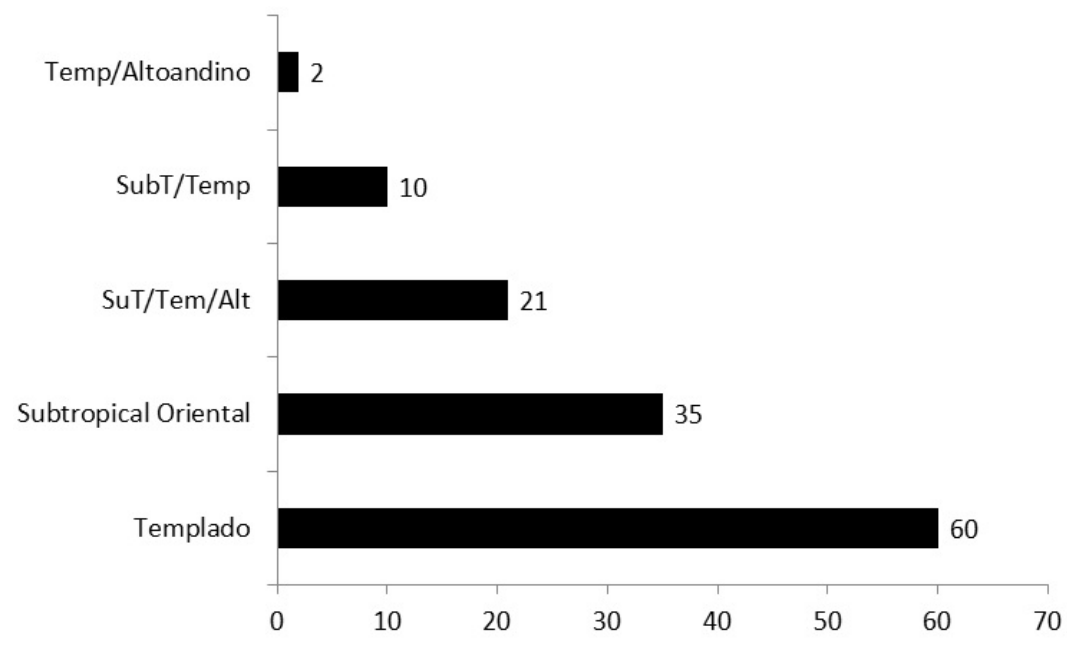

Figura 3. Número de publicaciones por piso zoogeográfico. Códigos: Temp/Altoandino: Templado/Altoandino, SubT/Temp: Subtropical Oriental/Templado, SubT/Tem/Alt: Subtropical Oriental/Templado/Altoandino.

\subsection{Temáticas de estudio}

$\mathrm{Al}$ analizar los estudios por el área de conocimiento investigada se definió que los temas de ecología $(n=51)$ y diversidad $(n=30)$ principalmente listados de especies, dominan sobre el resto. Se continúan en importancia publicaciones sobre distribución (registros inusuales y ampliaciones de rango de distribución) $(\mathrm{n}=18)$, conservación $(\mathrm{n}=15)$ y taxonomía $(n=13)$, y finalmente se puede mencionar un solo estudio sobre bioacústica, desarrollado en el área protegida (Flanagan, 1998) (Figura 4).
Ecuador se puede mencionar que el piso Templado (2000 a $3000 \mathrm{msnm}$ ) constituye la franja altitudinal más estudiada del área protegida con 60 investigaciones en total. Le sigue el piso subtropical oriental (1000 a $2000 \mathrm{msnm})$ con 35 investigaciones y 21 investigaciones abarcan los tres pisos zoogeográficos presentes en el parque (Templado, subtropical oriental y altoandino). Mientras que los pisos más elevados poseen 12 investigaciones en total, 10 que abarcan el piso subtemplado oriental y templado, $\mathrm{y}$ dos que abarcan los pisos templado y altoandino (Figura 3). (1) 
ción constituye el primer esfuerzo de este tipo desarrollado para un área protegida del país y propone lineamientos metodológicos (Figura 2) que pueden ser facilmente replicados en otras áreas protegidas, o incluso, en otros procesos o tipos de investigación. Por otra parte, es indiscutible la importancia que en la actualidad poseen los sistemas de información geográfica, entre otros elementos, puesto que estas herramientas permiten representar gráficamente los resultados de diversas investigaciones (Greene y Pick, 2012). Es por esto que, como uno de los pasos fundamentales del presente trabajo, se contempló el mapeo de la información generada con el propósito de evidenciar el grado de influencia territorial de las investigaciones recopiladas, los vacíos geográficos y temáticos sobre el conocimiento de los vertebrados existentes en el área protegida.

Sobre los grupos taxonómicos estudiados se puede acotar que la realidad encontrada es similar a la de otras latitudes. Es bien conocido que las aves y mamíferos se consideran los grupos taxonómicos me- jor conocidos del planeta (Stotz y col., 1996; Larsen y col., 2012), hecho también propuesto para Ecuador (Albuja y col., 2012). Este estudio corrobora esta tendencia y permite evidenciar que el grupo de vertebrados más estudiado del PNP corresponde a las aves $(50 \%$ de los estudios en el área protegida), seguido por investigaciones sobre los mamíferos $(20 \%)$ del sector (Figura 1a). El interés por la biodiversidad faunística existente en el territorio correspondiente al PNP posee una larga data, esto lo denotan la visita a esta región de un importante número de investigadores del American Museum of Natural History entre los años 1854 y 1920 (Chapman y col., 1926; Anthony, 1922). Sin embargo, luego de estos trabajos las investigaciones en esta zona fueron esporádicas, siendo retomadas a partir de los años setenta con las expediciones de Louisiana State University Museum of Zoology (LSUMZ); y con mayor fuerza a partir de finales de los años ochenta por parte de Zoological Museum of Copenhagen (ZMUC) (Bloch y col., 1991).

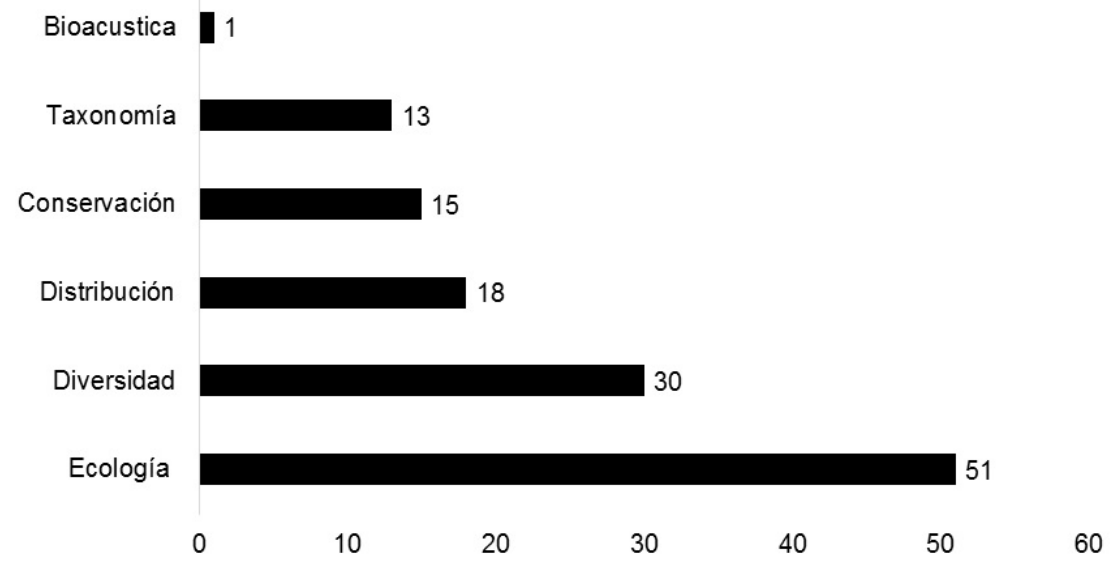

Figura 4. Número de publicaciones por temática de estudio sobre los vertebrados del Parque Nacional Podocarpus.

Estos trabajos cimentaron el interés por la zona en los años siguientes a la declaratoria del área protegida. Uno de los factores más influyentes para que este interés continúe hasta la actualidad, principalmente por la comunidad ornitológica, tiene relación con el descubrimiento de dos especies de aves en esta región en menos de una década $(1992,1997)$ (Krabbe y col., 1999; Coopmans y Krabbe, 2000). La Elaenia Tropandina (Myiopagis olallai) fue descubierta en el sector de Bombuscaro en 1992 (Coopmans y Krabbe, 2000) y la Grallaria Jocotoco (Gra- llaria ridgelyi) fue descubierta en 1997 en el sector de Tapichalaca al sur del PNP (Krabbe y col., 1999). Adicionalmente, especies como Cotinga Ventricastaña (Doliornis remseni) fueron registradas por primera vez para el país en el sector de Cajanuma en 1989 (Robbins, Rosenberg y Molina, 1994), todo esto en un territorio que no supera las 147000 ha.

Entre otros, es la importancia demostrada sobre la biodiversidad del sector la que influyó para que a finales de los años noventa la ONG Naturaleza Cultura Internacional establezca una estación científi- 
ca en el límite norte del área protegida, en la cual, con financiamiento de la Fundación Alemana para la Investigación Científica se ejecutan diversas acciones de investigación (Kiss y Bräuning, 2008), los mismos que se mantienen hasta la actualidad. Son los factores expuestos los que contribuyeron para que el número de investigaciones publicadas para el área protegida a partir de la década del año 1990 se incrementen de manera significativa. Aunque, en la actualidad (2011-2018), se ha experimentado un decrecimiento de al menos el $25 \%$ en este tipo de trabajos (Figura 1c).

Gran parte del flanco oriental del área de estudio lo constituyen bosques y páramos pristinos, estado atribuido a lo agreste y el dificil acceso a los mismos (Remache y col., 2004; Kiss y Bräuning, 2008), de ahí que aun persistan en el área protegida importantes zonas sin información sobre la riqueza biológica que albergan (Figura 1a). Albuja y col., (2012) proponen que la información sobre la fauna de Ecuador se encuentra dispersa y presenta dificultades en su acceso. El presente trabajo se desarrolla después de 35 años de la declaratoria oficial de esta área protegida y evidencia que existe un importante número de publicaciones en torno al objeto de estudio, pero estas en su mayoría están dispersas y no se encuentran disponibles para los actores institucionales vinculados a la gestión del área, principalmente para los tomadores de desiciones como el Ministerio del Ambiente de Ecuador.

El presente análisis permite definir algunas prioridades de investigación para el área protegida, tanto en el ámbito geográfico como temático. Los peces y reptiles del PNP requieren atención prioritaria, ya que poseen pocos estudios hasta el momento; además, es necesario incrementar el conocimiento sobre la biodiversidad y ecología de la fauna en el centro y sureste del parque. Los resultados evidencian que la mayoría de los estudios efectuados sobre los vertebrados del sector han sido desarrollados en sus bordes o su área de influencia inmediata, siendo el sistema lacustre Lagunas del Compadre el que cuenta con un estudio sobre los anfibios del sector. Extensos territorios internos del parque evidencian falencias en cuanto a investigacion, principalmente en el centro y sureste del área. Sin embargo, es conveniente señalar que el acceso a estos sitios es dificil, de ahí que para llenar estos vacíos de información se requerirán significativos esfuerzos de trabajo.

Por otra parte, temáticas que poseen significativa potencialidad de desarrollo en el sitio, como bio- acústica, deberían contar con nuevos esfuerzos de trabajo o sistematización. Esto por cuanto en la actualidad existen plataformas en línea (ej. XenoCanto, Internet Bird Collection) que albergan una importante colección de registros (fotográficos y auditivos) de aves de esta área protegida; sin embargo, este material no ha sido sistematizado o analizado en el contexto de aporte al área.

\section{Conclusiones}

El presente trabajo constituye una línea de base sobre las investigaciones de vertebrados efectuadas en este Parque Nacional, el mismo que permitirá priorizar y monitorear los futuros esfuerzos en esta temática para el sector. Si bien generalmente los análisis sistemáticos de información se enfocan en la recopilación y sistematización de la literatura existente en bases de datos científicas, este documento evidencia la importancia de incluir información gris de ámbito local para trabajos como el presente. Un 34\% $(n=44)$ de los estudios que componen los resultados de este trabajo corresponden a literatura gris, de ahí la importancia en la recopilación e inclusión de esta información en este tipo de revisiones.

La metodología propuesta constituye una valiosa oportunidad para entidades a cargo de una o más áreas protegidas, su aplicación es sencilla, de costo mínimo, y adaptable a diversas temáticas y realidades. La misma puede ser desarrollada por entidades estatales como el Ministerio del Ambiente, Gobiernos Autónomos Descentralizados o para áreas protegidas comunitarias o privadas. Es evidente que un claro conocimiento sobre los procesos de investigación efectuados en un sector determinado permitirá fortalecer la gestión del sitio.

\section{Referencias}

Aguirre, Z. y col. (2002). «Botánica Austroecuatoriana: Estudios sobre los Recursos Vegetales en las provincias de El Oro, Loja y Zamora Chinchipe». En: Ediciones Abya Yala, Quito. EC, 1-28. Online:https://bit.ly/2Ywe5wd.

Albuja, L. y col. (2012). Fauna de vertebrados del Ecuador. Escuela Politécnica Nacional. Online:https: / / bit.ly/2ZGLK2J.

Anthony, H. (1922). «Preliminary report on Ecuadorean mammals. No. 2». En: American Museum Novitates 32, 9. Online:https:/ / bit.ly/2yKLxzE. 
Apolo, W. (2002). «Botánica Austroecuatoriana. Estudios sobre los recursos vegetales en las provincias de El Oro, Loja y Zamora Chinchipe». En: ed. por Z. Aguirre, J. M. Madsen y H. Cotton E.and Balslev. Quito: Editorial Abya Yala. Cap. El Parque Nacional Podocarpus: Perspectivas de conservación y desarrollo, págs. 411-431.

Bloch, H. y col. (1991). "A survey of the montane forest avifauna of the Loja province, southern Ecuador». En: ICBP Study Reports 49, Online:https:/ / bit.ly/2ZGMtRv.

Calderón, S. (2002). «Botánica Austroecuatoriana Estudios sobre los recursos vegetales en las provincias de El Oro, Loja y Zamora-Chinchipe.» En: ed. por Z. Aguirre y col. Quito: Abya-Yala. Cap. El Parque Nacional Podocarpus y fundamentos legales que lo respaldan, págs. 433-451.

Chapman, F. y col. (1926). The distribution of bird-life in Ecuador: A contribution to a study of the origin of Andean bird-life. Order of the Trustees, American Museum of Natural History. Online:https: / / bit.ly/2KILDo0.

Constituyente, Asamblea Nacional, ed. (2008). Constitución Política de la República del Ecuador. Tribunal Constitucional de Ecuador. Online:https://bit.ly /2yOeU3L.

Coopmans, P. y N. Krabbe (2000). «A new species of flycatcher (Tyrannidae: Myiopagis) from eastern Ecuador and eastern Peru». En: The Wilson Bulletin 112.3, 305-313. Online:https: / / bit.ly / 2YSQv84.

Cuesta, F. y col. (2005). «Evaluación ecorregional de los páramos y bosques montanos de la Cordillera Real Oriental: componente terrestre. EcoCiencia». En: The Nature Conservancy. Quito, Ecuador, Online:https://bit.ly/2TvhNiP.

Davis, S.D. y col. (1997). Centres of Plant Diversity: A Guide and Strategy for Their Conservation. Ed. por S.D. Davis y col. Vol. 3: The Americas. Cambridge. Onlne: https: / / bit.ly / 2YUdVu2. IUCN Publications Unit.

Dinerstein, E. y col. (1995). A conservation assessment of the terrestrial ecoregions of Latin America and the Caribbean. The World Bank. Online:https:/ / bit. ly/2TvBYwU.

Dudley, N. y S. Stolton (2010). Arguments for protected areas: multiple benefits for conservation and use. Routledge. Online:https:/ / bit.ly/2GSW0xK.

Duellman, W. (1979). «The herpetofauna of the Andes: patterns of distribution, origin, differentiation, and present communities». En: The South
American herpetofauna: its origin, evolution, and dispersal 7, 371-459. Online:https : / / bit . ly / 2M4Mn3f.

Flanagan, J., ed. (1998). Aves de Cajanuma: Parque Nacional Podocarpus. Vocalizaciones de algunas especies del bosque nublado montañoso tropical. LojaEcuador. Online: https:/ / bit.ly/2M7Rboy : Fundación Ecológica Arcoíris.

Freile, J. F. y R. Restall (2018). Birds of Ecuador. Helm, Bloomsbury Publishing Plc. Online:https: / / bit. ly/2OOoBum.

Greene, R. y J. Pick (2012). Exploring the urban community: A GIS approach. Prentice Hall. Online:https:/ / bit.ly/2yM1XrI.

Jiménez-Prado, P. (2010). «Fauna de Vertebrados del Ecuador». En: ed. por C. Boada y col. LojaEcuador: Universidad Técnica Particular de Loja. Cap. Peces de Ecuador, págs. 17-94.

Kiss, K. y A. Bräuning (2008). El bosque húmedo de montaña: investigaciones sobre la diversidad de un ecosistema de montaña en el Sur del Ecuador. Deutsche Forschungsgemeinschaft. Online:https: / / bit.ly/2SGn1Z0.

Krabbe, N. y col. (1999). "A new species of antpitta (Formicariidae: Grallaria) from the southern Ecuadorian Andes». En: The Auk 116.4, 882-890. Online:https:/ / bit.ly/2GUqM9n.

Larsen, F. y col. (2012). «Birds as biodiversity surrogates: will supplementing birds with other taxa improve effectiveness?» En: Journal of Applied Ecology 49.2, 349-356. Online:https: / / bit.ly / 2YRHQ63.

Maldonado, N. y P. Numa (2002). "Clima y vegetación de la región sur del Ecuador». En: Aguirre J, Madsen JE, Cotton E, Balslev, editores. Botánica Austroecuatoriana. Quito: Ediciones Abya Ayala, págs. 1-28.

Manterola, C., P. Astudillo y E. Arias (2013). «Revisiones sistemáticas de la literatura. Qué se debe saber acerca de ellas». En: Cirugía Española 91.3, 149-155. Online:https://bit.ly/2TqxA3L.

Medina-López, C., J. Marín-García y R. AlfallaLuque (2010). «Una propuesta metodológica para la realización de búsquedas sistemáticas de bibliografía (A methodological proposal for the systematic literature review)». En: WPOMWorking Papers on Operations Management 1.2, 13-30. Online:https:/ / bit.ly/2M86Dkr.

Ministerio de Agricultura, Ganadería y Pesca de Ecuador (1983). «Declaratoria del Parque Nacional Podocarpus. Acuerdo Ministerial $N^{\circ}$ 0398, 
Registro Oficial. $N_{0} 404,5$ de enero de 1983». En: Registro Oficial de Ecuador. Quito.

Mittermeier, R. (1997). Megadiversity: Earth's biologically wealthiest nations. Agrupacion Sierra Madre. Online:https://bit.ly/2yMlZm4.

Myers, N. y col. (2000). «Biodiversity hotspots for conservation priorities». En: Nature 403.6772, 853. Online:https://go.nature.com/2Gnztdw.

Neill, D. (2012). « ¿ Cuántas especies nativas de plantas vasculares hay en Ecuador?» En: Revista Amazónica Ciencia y Tecnología 1.1, 70-83. Online:https://bit.ly/2Rwi0VH.

Ordóñez-Delgado, L. (2011). Caracterización biofísica de los sistemas lacustres del Parque Nacional Podocarpus y Parque Nacional Yacuri, Andes Tropicales del Sur del Ecuador. Inf. téc. Loja-Ecuador. Online:https:/ / bit.ly/2MeoINV: Ministerio del Ambiente de Ecuador (Zonal 7).

Primack, R. y col. (1998). Fundamentos de conservación biológica. Perspectivas latinoamericanas. Ed. por R. Primack y col. México: Fondo de Cultura Económica.

QGIS, Development Team (2018). QGIS geographic information system. Inf. téc. Open Source Geospatial Foundation Project.

Remache, G. y col. (2004). Integridad Ecológica del Microcorredor de Conservación Yacuambi-PodocarpusSabanilla. EcoCiencia. Grupo de Trabajo de Páramos de Loja-GTPL. Fundación Arcoiris.

Robbins, M., G. Rosenberg y F. Molina (1994). «A new species of cotinga (Cotingidae: Doliornis) from the Ecuadorian Andes, with comments on plumage sequences in Doliornis and Ampelion». En: The Auk 111.1, 1-7. Online:https://bit. ly/2R9P8mF.

Ron, S. y col. (2019). «Anfibios del Ecuador». En: Museo de Zoología, Pontificia Universidad Católica Del Ecuador, Online:https:/ / bit.ly/2s6zWHu.
Rueda, M. (2003). «Presentación de la colección. La investigación educativa en México, 1992-2002». En: 2003). Consejo Mexicano de Investigación Educativa A. C. 1992-2002. Online:https: / / bit.ly / 2DX8GUn.

Serrano, F. (2008). «Reserva de biósfera PodocarpusEl Condor: Reflexiones del primer año de gestión». En: XXXII Jornadas Nacionales de Biología ]. Universidad Técnica Particular de Loja. Online:https://bit.ly/2yNwm8W.

Stotz, D. y col. (1996). Neotropical birds: ecology and conservation. University of Chicago Press. Online:https://bit.ly/2YBHxRF.

Terborgh, J. y B. Winter (1983). «A method for siting parks and reserves with special reference to Columbia and Ecuador». En: Biological Conservation 27.1, 45-58. Online:https:/ / bit.ly/2GSXZlm.

Tirira, D. (2018). Mamíferos del Ecuador: Lista actualizada de especies/Mammals of Ecuador: Updated checklist species. Versión 2017.2. Inf. téc. Online: https: / / bit.ly/2VtvcJU: Fundación Mamíferos y Conservación.

Torres-Carvajal, O., G. Pazmiño-Otamendi y D. Salazar-Valenzuela (2018). Reptiles del Ecuador. Versión 2018.0. Español. Museo de Zoología, Pontificia Universidad Católica del Ecuador.

UICN (2014). Lista Roja de Especies Amenazadas de la UICN. Resumen para América del Sur. UICN oficina Regional para América del Sur.

Urrútia, G. y X. Bonfill (2010). «Declaración PRISMA: una propuesta para mejorar la publicación de revisiones sistemáticas y metaanálisis». En: Medicina clínica 135.11, 507-511. Online:https: / / bit.ly/2GPIc75.

Watson, J., D. Dudley N. Segan y M. Hockings (2014). «The performance and potential of protected areas». En: Nature 515.7525, 67. Online:https://go.nature.com/2t9QDpu. 